\title{
Spontaneous massive haemothorax in von Recklinghausen's disease
}

\author{
AJ LARRIEU, SA HASHIMOTO, PETER ALLEN \\ From the Division of Cardiovascular and Thoracic Surgery, Department of Surgery and Division of Neurology, \\ Department of Medicine, University of British Columbia, Vancouver, Canada
}

Spontaneous massive intrathoracic bleeding is infrequent and usually secondary to pulmonary infarction, arteriovenous fistula, neoplasm, ruptured aortic aneurysm, rupture of pleural adhesions, and, rarely, endometriosis of the pleura. ${ }^{1}$

We wish to document another cause of spontaneous massive haemothorax in a patient with disseminated neurofibromatosis (von Recklinghausen's disease). Bleeding was from a vascular Schwannoma in the right posterior mediastinum. There is only one other published comparable patient with this type of exsanguinating haemothorax. ${ }^{2}$

\section{Case report}

A 44-year-old white woman with von Recklinghausen's disease since the age of 8 years was admitted to hospital after several grand mal seizures.

She had hada left thoracotomy in 1966 for excision of an upper posterior mediastinal benign neurofibroma. In 1978 , the patient developed fits, but extensive neurological investigation was negative. There was no family history of fits. She progressed well on anticonvulsant therapy until six days before the haemothorax when she was again admitted to hospital for further neurological investigation.

On examination, the patient, who was in a post-ictal state, showed widely disseminated neurofibromata and café-au-lait spots. Blood pressure was $100 / 60$, pulse $80 / \mathrm{min}$ and regular, respirations 20 and slightly laboured. Chest examination revealed thoracic scoliosis, clear breath sounds, and a 2/6 systolic ejection murmur over the aortic area. Neurological examination revealed a drowsy female with no spontaneous speech. Power was decreased in the right side compatible with a Todd's palsy. There was no other neurological abnormality. Laboratory examinations were unremarkable. No chest radiograph was performed, but a thoracic spine film revealed mild scoliosis.

The patient improved but on the sixth day developed sudden sharp epigastric and right lower costal pain that increased on inspiration. Abdominal examination was negative. Within two hours the pain increased in severity and had localised to the right anterior chest

Address for reprint requests: Dr Peter Allen, Division of Cardiovascular and Thoracic Surgery, University of British Columbia, 700 West 10th Ave, Vancouver, BC, Canada. and back. Chest radiography showed a large right pleural effusion. Thoracentesis revealed bright blood, and through an intercostal tube $1800 \mathrm{ml}$ was removed. Shortly after she became shocked with a blood pressure of $60 \mathrm{~mm} \mathrm{Hg}$ and pulse of $120 / \mathrm{min}$. She was resuscitated by rapid infusion of three units of blood and two units of colloid.

At an emergency operation 3.51 of blood and clot were removed from the right chest through a right posterolateral thoracotomy. The source of bleeding was from an intercostal artery and vein which penetrated a sessile gelatinous $5 \times 2 \mathrm{~cm}$ greyish tumour adjacent to the ninth dorsal vertebra (fig 1). Bleeding was controlled by

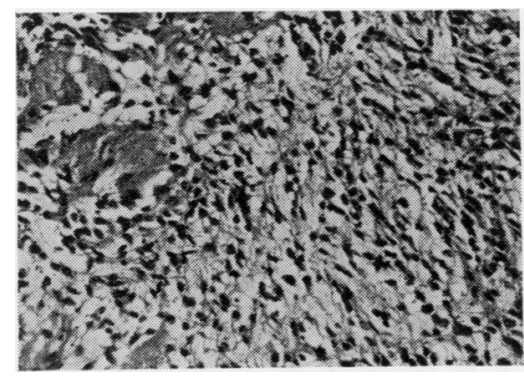

Fig 1 Photomicrograph of benign Schwannoma. (Haematoxylin and eosin $\times 250$ ).

removing the anterior part of the tumour to expose the bleeding vessels before suturing. The area occupied by the tumour was marked with metal clips (fig 2 and 3). After the operation she improved quickly and was discharged eight days later. Histological examination of the friable tumour revealed a benign Schwannoma with numerous dilated blood vessels (fig 1).

\section{Discussion}

Von Recklinghausen's neurofibromatosis is a wellrecognised entity characterised by abnormal cutaneous pigmentation and multiple skin tumours. It is a hereditary disorder originating in the neuroectoderm and mesoderm and although transmitted by a dominant gene, it is expressed in about $80 \%$ of those who have the gene. ${ }^{1}$

The patient with von Recklinghausen's disease requires 


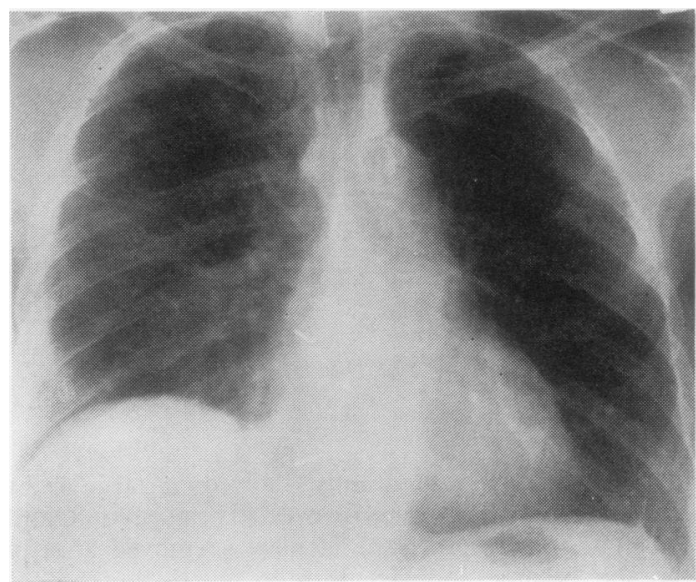

Fig 2 Posteroanterior chest radiograph after operation showing the location of the tumour marked by metal clips.

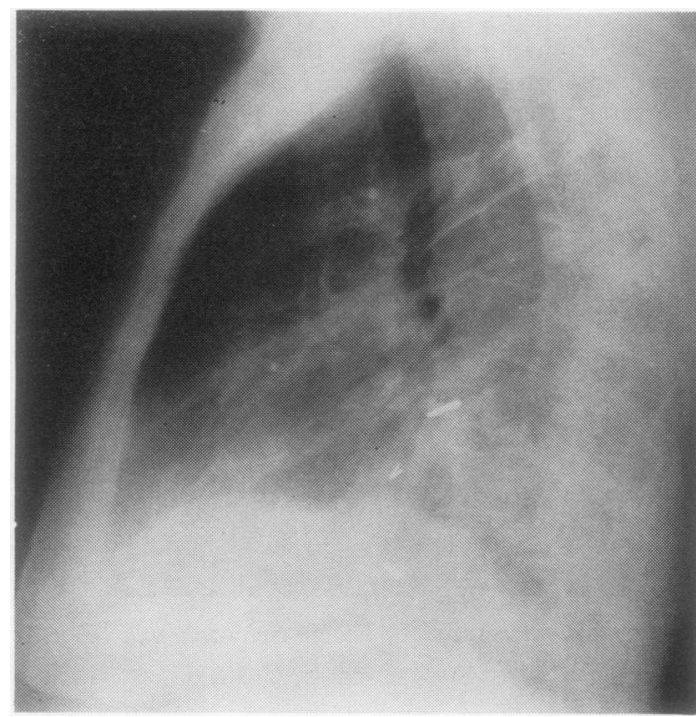

Fig 3 Lateral view of the tumour sites.

special attention because of many unique problems. Involvement of the CNS is almost always present, ranging from epilepsy, as in our patient, to the development of acoustic neuromas, gliomas, and meningiomas. Associ- ation with pheochromocytoma and other syndromes and an increased incidence of malignant tumours are further reasons for close follow-up. ${ }^{1}$

Bleeding from these tumours is a rare but potentially lethal complication. Bleeding from neurofibromas of the alimentary tract, chiefly the jejunum, has been reported and attributed to their hypervascularity. ${ }^{3}$ In some of these patients large segments of jejunum were excised to control bleeding. Bleeding into bone with the formation of cysts has been reported, as well as subarachnoid haemorrhage from acoustic neuromas. ${ }^{4}$

Butchart et al reported a patient with neurofibromatosis who bled extensively into the right haemothorax after spontaneous rupture of an intercostal artery. The rupture was attributed to microvascular lesions seen in neurofibromatosis which affect arteries $0.5-1 \mathrm{~mm}$ in diameter, producing fibrous intimal thickening and microaneurysms that may rupture. ${ }^{5}$

In our patient, bleeding occurred from an intercostal artery and vein in the base of a $5 \times 2 \mathrm{~cm}$ soft vascular Schwannoma. Because of tissue necrosis no vessel was seen in the tumour on histological study. Therefore, it is difficult to say whether bleeding was caused by micro-aneurysmal dilatation or erosion of the intercostal vessels in the tumour. The latter is more likely. Regardless of the mechanism of bleeding in spontaneous haemothorax or bleeding into other organs associated with neurofibromatosis, prompt, vigorous treatment may be lifesaving. Consequently, family physicians, neurologists, and thoracic surgeons should be aware that spontaneous massive haemothorax in a patient with von Recklinghausen's disease may occur from erosion of thoracic vessels by tumour or from degeneration of a hypervascular neural tumour.

\section{References}

1 Wander JV, Das Gupta TK. Neurofibromatosis. Curr Prob Surg 1977;14:1-81.

${ }^{2}$ Butchart EG, Grotte GH, Barnsky WC. Spontaneous rupture of an intercostal artery in a patient with neurofibromatosis and scoliosis. J Thorax Cardiovasc Surg 1975;69:919-21.

${ }^{3}$ Brasfield RD, Das Gupta TK. Von Recklinghausen's disease: clinicopathological study. Ann Surg 1972;175: 86-94.

${ }^{4}$ Gleeson RK, Butzer JF, Grin OD. Acoustic neurinoma presenting as subarachnoid haemorrhage. $J$ Neurosurg $1978 ; 49: 602-4$.

${ }^{5}$ Salyer WR, Salyer DC. The vascular lesion of neurofibromatosis. Angiology 1974;25:510-9. 